\title{
Differential expression of two somatostatin receptor subtype 1 mRNAs in rainbow trout (Oncorhynchus mykiss)
}

\author{
B J Slagter and M A Sheridan
}

Department of Biological Sciences and Regulatory Biosciences Center, North Dakota State University, Fargo, North Dakota 58105, USA

(Requests for offprints should be addressed to M A Sheridan, Department of Biological Sciences, North Dakota State University, Fargo, North Dakota 58105, USA; Email: Mark.Sheridan@ndsu.nodak.edu)

\begin{abstract}
Somatostatins (SSs) play important roles in the growth, development and metabolism of vertebrates. In this study, cDNAs for two unique somatostatin receptor variants were cloned and sequenced from rainbow trout. The two cDNAs, one consisting of $1755 \mathrm{bp}$ and the other of $1743 \mathrm{bp}$, share $63.6 \%$ identity in nucleotide sequence and $94.1 \%$ identity in deduced amino acid sequence and presumably arose through gene duplication. Each cDNA encodes for a putative 371-amino acid somatostatin receptor (one designated sst1A and the other sst1B) containing seven transmembrane domains. Rainbow trout sst1A and sst1B have 64.4 and $65.5 \%$ similarity respectively with human sst1 and only $43-60 \%$ similarity with other subtypes.

Trout sst1 mRNAs are differentially expressed, both in terms of distribution among tissues as well as in terms of abundance within selected tissues. Both sst1A and sst1B mRNAs were present in brain, stomach, liver, pancreas, upper and lower intestine, pyloric cecum, kidney and muscle, whereas only sst1B mRNA was present in the esophagus. sst1A mRNA was more abundant than sst1B in the optic tectum, whereas sst1B mRNA was more abundant than sst1A in liver. sst1A and sst1B mRNAs were equally abundant in pancreas. These findings contribute to the understanding of the evolution of the SS signaling system and provide insight into the mechanisms that regulate the expression of SS receptors.
\end{abstract}

Journal of Molecular Endocrinology (2004) 32, 165-177

\section{Introduction}

Somatostatins $(\mathrm{SSs})$ are a relatively diverse family of peptide hormones that regulate a wide range of physiological processes in vertebrates, including the modulation of growth, development and metabolism (Sheridan et al. 2000). The multifunctional nature of this peptide hormone family arises from an elaborate, multifaceted signaling system that consists of numerous forms of SS molecules that are capable of binding to a variety of receptors. The molecular heterogeneity of the SS family arises from tissue-specific variations in biosynthesis from larger precursor molecules as well as from the existence of multiple SS genes. The different forms of SS seen in mammals such as SS-25 or SS-28 are
N-terminal extensions of SS-14 and result from differential processing of the same precursor, preprosomatostatin I (PPSS I) (Conlon 1989). Several non-mammalian species, including teleost fish, possess multiple PPSSs, each capable of being processed to an SS isoform (Conlon et al. 1997, Sheridan et al. 2000, Lin \& Peter 2001). Rainbow trout, for example, possess a PPSS I that contains the highly conserved SS-14 at its C-terminus (Kittilson et al. 1999) as well as two different PPSS IIs, each containing $\left[\mathrm{Tyr}^{7}, \mathrm{Gly}^{10}\right]$-SS-14 at their C-termini (Moore et al. 1999).

Somatostatins exert their actions through binding to specific plasma membrane receptors. Since 1992, five somatostatin receptor (sst) subtypes, sst 1-sst5, have been identified by molecular cloning 


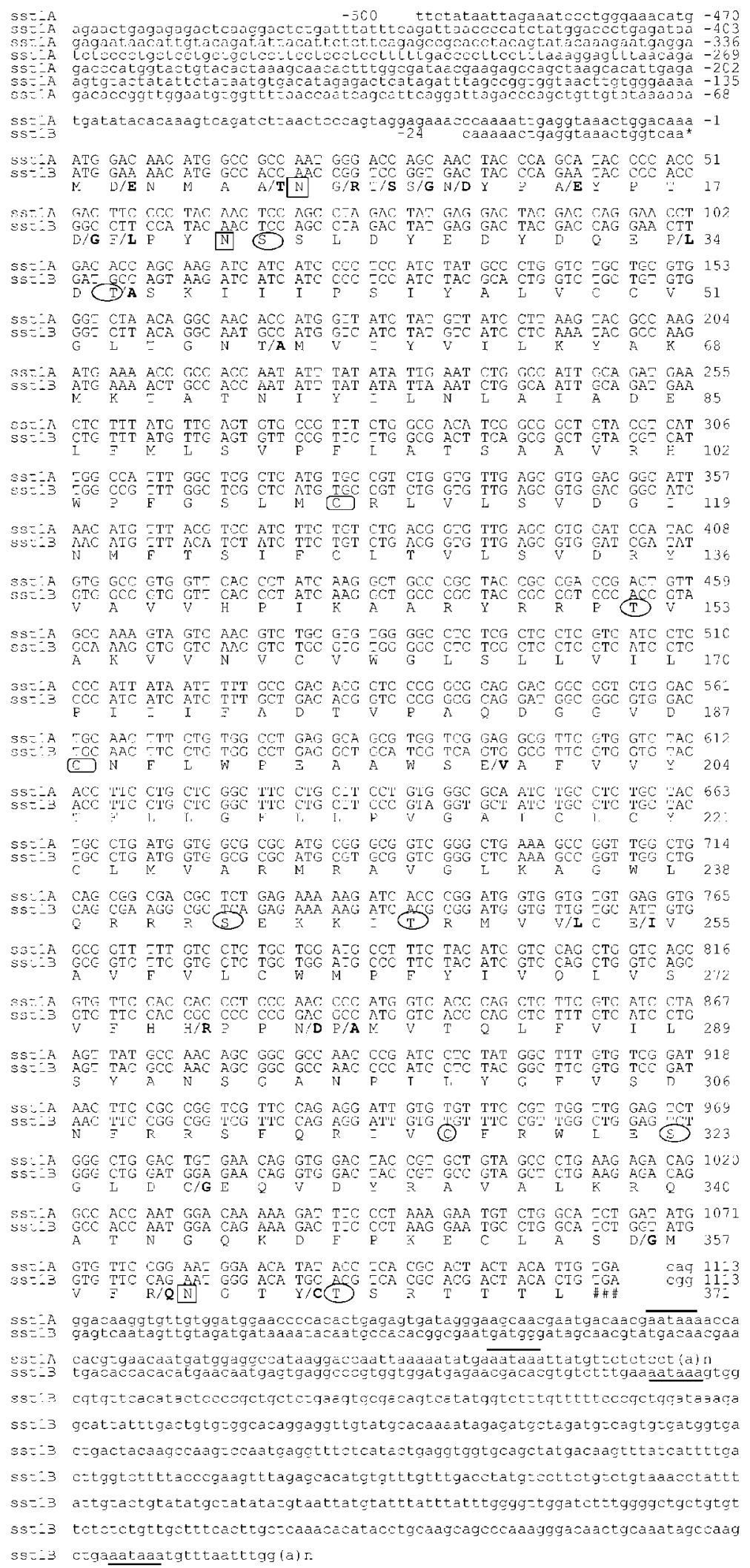


of their cDNAs or genes in mammals (Hoyer et al. 1995).

The ssts are seven transmembrane domain (TMD) guanine nucleotide binding ( $\mathrm{G})$-proteincoupled receptors; the receptors display subtypespecific selective binding to ligands (Reisine \& Bell 1995, Patel \& Srikant 1997, Meyerhof 1998). Although SS binding in non-mammalian species has been known for some time (Pesek \& Sheridan 1996), details regarding the structural characterization of ssts is just emerging (Lin \& Peter 2001). In this study, we used rainbow trout to further understand the polygenic origins of ssts and to provide insight into mechanisms which may serve to regulate their expression.

\section{Materials and methods}

\section{Experimental animals}

Juvenile rainbow trout of both sexes were obtained from Dakota Trout Ranch near Carrington, ND, USA and were transported to North Dakota State University. They were maintained in well-aerated, 800 l circular tanks supplied with recirculated $(10 \%$ make-up volume per day) dechlorinated municipal water at $14{ }^{\circ} \mathrm{C}$ under a $12 \mathrm{~h}$ light: $12 \mathrm{~h}$ darkness photoperiod. Fish were fed to satiety twice daily with PMI AquaMax Grower (Brentwood, MO, USA), except for 24-36 h before experiments. Animals were acclimated to laboratory conditions for at least 4 weeks prior to experimentation.

\section{RNA extraction}

The fish were anaesthetized in $0.05 \%$ 2phenoxyethanol. Selected tissues (e.g. brain, Brockmann body, etc.) were removed, placed in $2 \mathrm{ml}$ microcentrifuge tubes, and immediately frozen on dry-ice. Total RNA was extracted using TRI Reagent (Molecular Research Center, Inc.,
Cincinnati, OH, USA) as specified by the manufacturer. Each RNA pellet was redissolved in $75 \mu \mathrm{l}$ RNase-free deionized water and quantified by u.v. $A_{260}$ spectrophotometry. RNA samples were then stored at $-90{ }^{\circ} \mathrm{C}$ until further use. Brain and Brockmann bodies (endocrine pancreas) were selected as source material for sequence analysis because of the central importance of these organs to the function of animals and because they are known target organs of SS in fish and mammals (Sheridan et al. 2000)

\section{Oligonucleotide primers and probes}

Gene-specific oligonucleotide primers and probes were custom synthesized by Sigma-Genosys (The Woodlands, TX, USA) or supplied with $3^{\prime}$ - and 5'-RACE kits (Gibco/BRL, Gaithersburg, MD, USA). Primers were used for reverse transcription (RT) and PCR without further purification. Oligonucleotides were $5^{\prime}$-end labeled with $\gamma$ $\left[{ }^{32} \mathrm{P}\right]$ ATP (Amersham) using T4-polynucleotide kinase (Promega) (Sambrook et al. 1989). The full-length $\gamma$-actin probe (human fibroblast; Gunning et al. 1983) was labeled with $\left[{ }^{32} \mathrm{P}\right] \mathrm{CTP}$ by random priming (cf. Promega). All radiolabeled probes were purified over Elutip-D columns (Schleicher and Schuell, Keene, NH, USA) as detailed by the supplier.

\section{Isolation and sequence of somatostatin receptor cDNAs}

A three-phase approach was adopted for the isolation of selected cDNAs using RT-PCR and rapid amplification of cDNA ends (RACE)-PCR. In phase I, endogenous poly $(\mathrm{A})^{+} \mathrm{RNA}$ was reverse transcribed from $1 \mu \mathrm{g}$ trout pancreatic and brain total RNA with Superscript II reverse transcriptase and a 37-nucleotide antisense adapter primer

Figure 1 Comparison of the cDNA nucleotide and deduced amino acid sequences of rainbow trout sst1A and sst1B. Nucleotides are numbered from $5^{\prime}$ to $3^{\prime}$ and the amino acid residues (denoted using single-letter abbreviations) are numbered with the start codon in the open reading frame. A gap (indicated by asterisk) has been inserted just prior to the start codon for maximum alignment of the $5^{\prime}$ untranslated region. The complete amino acid sequence for sst1 $\mathrm{A}$ is shown. Amino acid residues of the corresponding sst1B that differ from those of sst1 A are shown in boldface type following sst1A residues with a slash. The stop sequence is denoted by '\#\#\#'. Putative polyadenylation signals are overlined for sst1A and underlined for sst1B. Potential N-linked glycosylation sites are denoted by a $\square$ around the amino acid. A potential site for a palmitoyl anchor is denoted by a $\bigcirc$ around the amino acid. Amino acids that potentially are associated with a disulfide bond are surrounded by a rectangle with rounded corners. Potential phosphorylation sites are denoted with a oval around the amino acid. 


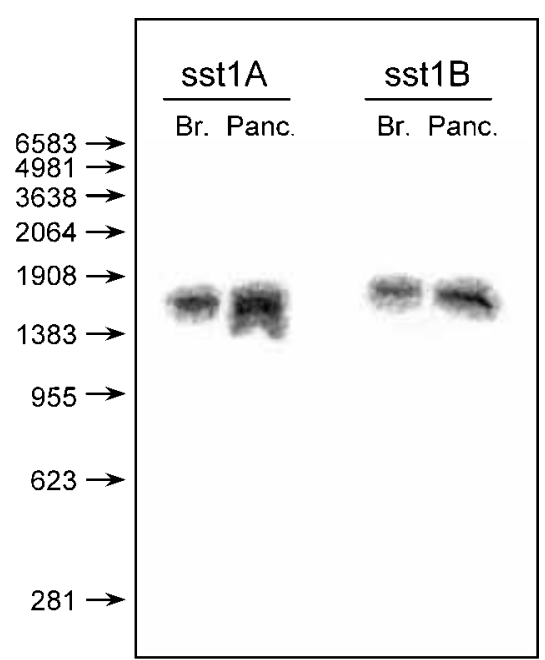

Figure 2 Northern blot analysis of poly $(A)^{+}$RNA reveals single transcripts for rainbow trout sst $1 \mathrm{~A}$ and sst1B. Approximately $500 \mathrm{ng}$ of poly $(\mathrm{A})^{+}$RNA per lane was size fractionated, electroblotted to a nylon membrane, and then hybridized with gene-specific radiolabeled oligonucleotide probes. Blots were visualized with a Cyclone phosphor imaging system on a type "ST" (supersensitive) screen. BR, brain; Panc., pancreas.

containing 17 thymine bases at its $3^{\prime}$ end (Gibco/BRL technical bulletin). Five microliters of the RT reaction were used as template for PCR with a 20-base forward SS receptor gene-specific primer (GSP-1; 5'-GACGGITACSTGGCYGTG GT-3') and a 23-base reverse SS receptor gene-specific primer (GSP-2; 5'-AGGATRGGG TTGGCRGAGCTGTT-3'). Degenerate GSP-1 and GSP-2 primers were designed from previously known mammalian SS receptor sequences in transmembrane domain regions that contained highly conserved nucleotide sequences. Following an initial denaturation cycle of $94^{\circ} \mathrm{C}$ for 5 min, 35 PCR cycles were performed, each consisting of $1 \mathrm{~min}$ denaturation $\left(94^{\circ} \mathrm{C}\right), 1 \mathrm{~min}$ annealing $\left(42^{\circ} \mathrm{C}\right.$ ) and $1 \mathrm{~min}$ extension $\left(72^{\circ} \mathrm{C}\right)$. In the last cycle, the extension time was increased to $10 \mathrm{~min}$ to ensure complete extension. The resulting PCR product was visualized by electrophoresis on an agarose gel containing 1\% (w/v) OmniPur agarose (EM Science, Gibbstown, NJ, USA) and $1 \%$ (w/v) NuSieve GTG agarose (BioWhittaker Molecular Applications, Rockland, ME, USA) in $1 \times$ Tris-borate-EDTA $(\mathrm{TBE})$ buffer followed by ethidium bromide staining and u.v. transillumination. Amplified fragments were directly cloned into the pGEM-T Easy Vector (Promega). Positive
A
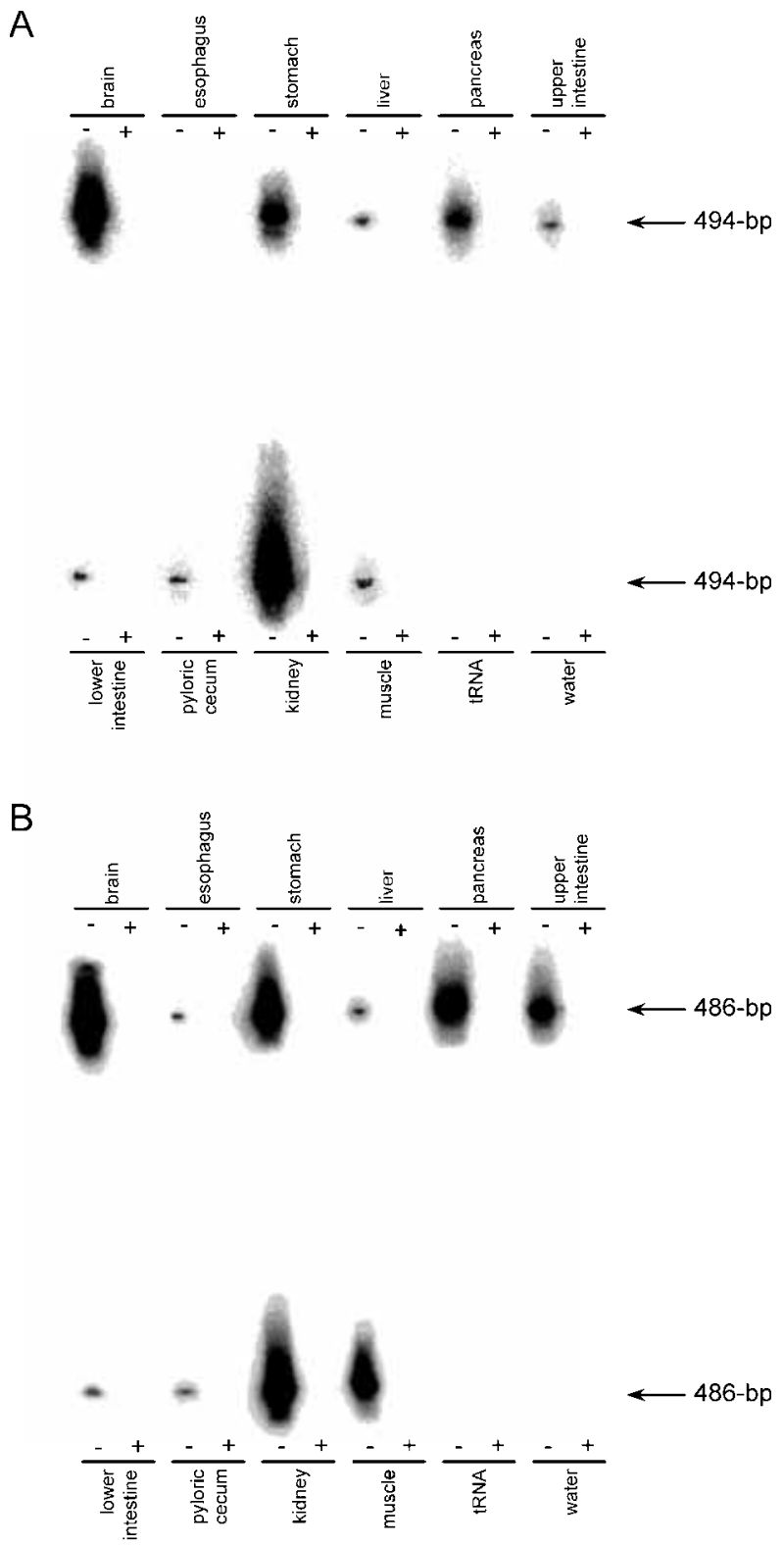

Figure 3 Expression of sst1A (A) and sst1B (B) mRNAs in various tissues of rainbow trout. Total RNA was treated with (+) or without (-) RNase and then subjected to RNA template-specific (RS)-PCR. Resulting products were analyzed by Southern blot hybridization using gene-specific ${ }^{32} \mathrm{P}$-labeled oligonucleotide probes. The size of the RS-PCR products matched the size (494 and 486 bp for sst1A and sst1B respectively) predicted from cDNA sequences and primer locations.

colonies were identified by agarose gel electrophoresis of restriction enzyme digests (EcoRI; Promega) of purified plasmid preparations (Ahn et al. 2000). Plasmid DNA (75 fmol) was sequenced 


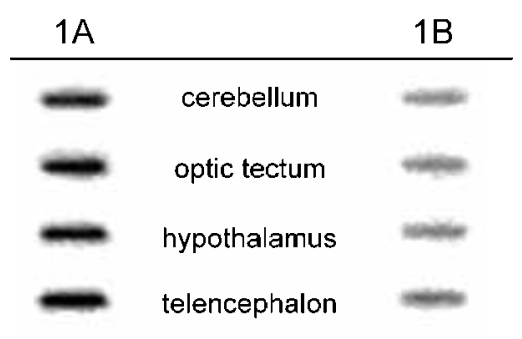

Figure 4 Northern slot-blot analysis of total RNA isolated from various regions of the brain. Approximately $10 \mu \mathrm{g}$ of total RNA were slotted in duplicate directly onto nylon membrane, hybridized with radiolabeled sst1A- or sst1B-specific oligonucleotide probes and visualized with a Cyclone phosphor imaging system.

with the CEQ 2000 sequencer using the Dye Terminator Cycle Sequencing Quick Start Kit (Beckman Coulter, Fullerton, CA, USA) according to the manufacturer's protocol.

In phase II, isolation of the $3^{\prime}$ cDNA sequence was accomplished by 3'-RAGE PGR (Gibco/BRL technical bulletin). Endogenous poly(A) ${ }^{+}$RNA was again reverse transcribed as described previously; $5 \mu \mathrm{l}$ of the RT reaction was used as template for 3'-RACE PCR with a 23-base sst gene-specific primer (GSP-3; 5'-TGCCCTTCTACATCGTCG AGCTG-3') and the universal amplification primer (Gibco/BRL). PCR was performed as in phase I. The amplified product was identified, cloned and sequenced as described previously.

In phase III, isolation of the $5^{\prime}$ cDNA sequence was accomplished by 5'-RACE PGR (Gibco/BRL technical bulletin). Somatostatin receptor mRNA was exclusively reverse transcribed from pancreatic and brain total RNA using GSP-2. The RT reaction conditions were the same as those described previously. The resulting cDNA was purified twice over a Glass Max spin column (Gibco/BRL) to remove unincorporated dNTPs and primer and eluted with $50 \mu$ sterile water. One-fifth of the purified cDNA was tailed at the $3^{\prime}$ end with dCTP using the enzyme terminal deoxynucleotidal transferase (Gibco/BRL); $5 \mu \mathrm{l}$ of the tailing reaction mixture was used as template for 5'-RACE PGR with sst GSP-4 (5'-GCGGT AGCGGGCAGCCTTGA-3') and anchor primer (Gibco/BRL). Thirty-five PCR cycles were performed as described in phase $\mathrm{I}$, except that the reaction mixture was heated to $94{ }^{\circ} \mathrm{C}$ for $4.5 \mathrm{~min}$ prior to the addition of Taq DNA polymerase (Qiagen, Chatsworth, CA, USA). The amplified

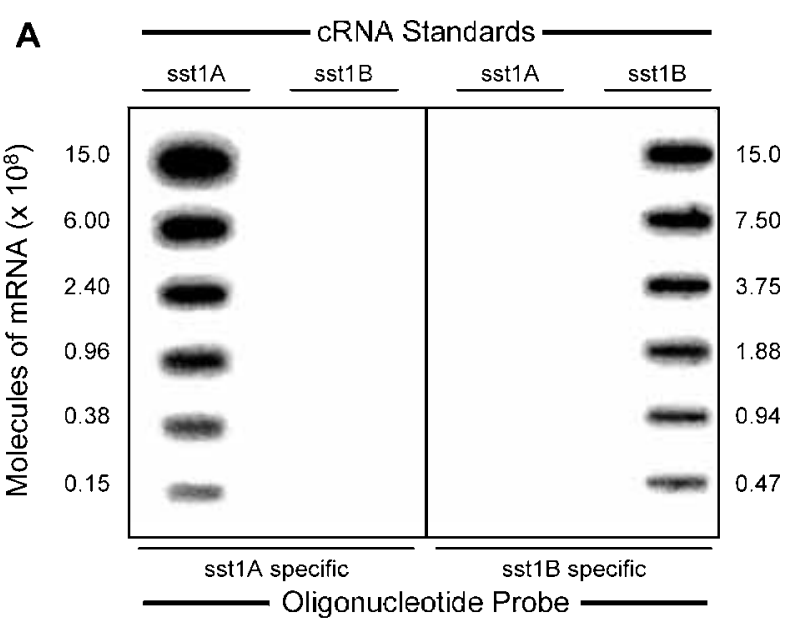

B

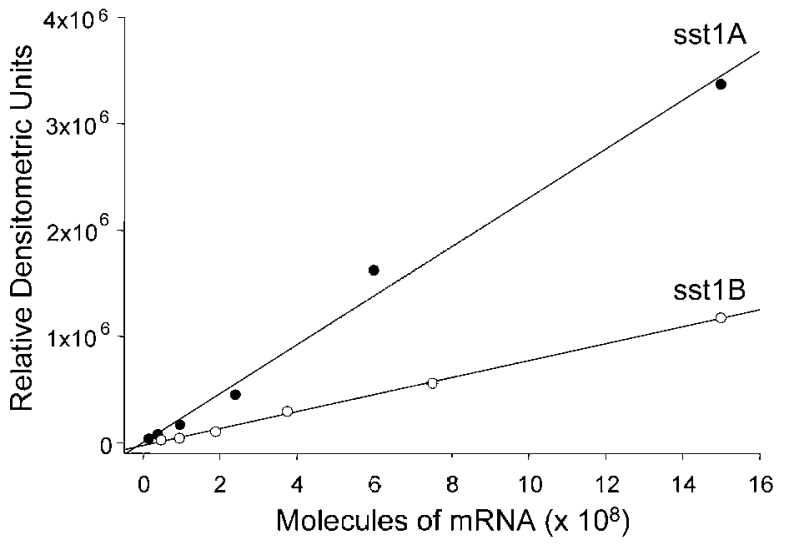

Figure 5 Quantitative slot-blot analysis for sst1 $\mathrm{A}$ and sst1B mRNAs. Sample total RNA (c. $10 \mu \mathrm{g})$ and serial dilutions of in vitro-synthesized cRNA standards for sst1A and sst1B (A) were slotted in duplicate directly onto nylon membrane, hybridized with radiolabeled sst1A- or sst1B-specific oligonucleotide probes and visualized with a Cyclone phosphor imaging system. Sample RNA was quantitated after correction for background by relation to standards plotted as a function of the number of sst1A or sst1B molecules per microgram of total RNA(B).

product was visualized by agarose gel electrophoresis, cloned and sequenced as described previously.

\section{Analysis of RNA and DNA}

Northern blot analysis (Moore et al. 1999) was performed to evaluate the number and size of transcripts as well as to verify that the gene-specific 


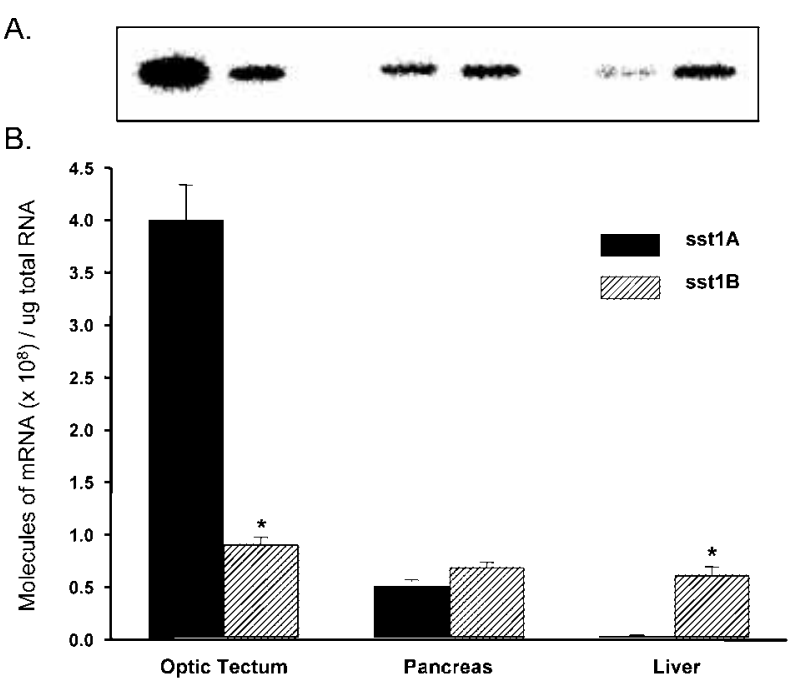

Figure 6 Relative abundance of sst1A and sst1B mRNAs in the optic tectum, pancreas and liver of rainbow trout. Sample total RNA was subjected to slot-blot quantitation as shown in Fig. 5. Data are presented as $(A)$ representative slot blots and $(B)$ means \pm S.E.M. $(n=8) ;$ * Significant difference compared with the sst1A group within the same tissue $(P<0.05)$.

oligonucleotide radiolabeled probes $(\operatorname{sst} 1 \mathrm{~A}(\mathrm{as})$ : 5'-GACAAAAACGGCGACGTCACACAC-3'; or sst1B(as): 5'-CACGAAGACGGCGACAATGCAC AA- $3^{\prime}$ ) hybridized only with the respective sstlA and sst1B transcripts in poly $(\mathrm{A})^{+}$RNA extracted from the brain and pancreas of rainbow trout.

RNA template-specific PGR (RS-PCR) was used to qualitatively evaluate the expression of sstlA and sst1B mRNAs among various tissues because of its high specificity (amplification of false positives derived from contaminating genomic DNA is excluded) and high sensitivity (Shuldiner et al. 1991). A d ${ }_{26} t_{30}$ primer (5'-CATGTACGTTGATC AACGGTCTCGTGGCAGAGGATRGGGTTG GCRCAGCTGTTRGC-3'), containing 26 bases at its $3^{\prime}$ end complementary to both sst $1 \mathrm{~A}$ and sst1B $\left(\mathrm{d}_{26}\right)$ and 30 bases of non-specific tagging sequence at its $5^{\prime}$ end $\left(t_{30}\right)$, was used to co-reverse transcribe sst1A and sst1B mRNA in total RNA $(c .15 \mu \mathrm{g})$ isolated from tissues. Five microliters of the $\mathrm{RT}$ reaction were incubated with or without RNase-A and subjected to PGR as previously described (Moore et al. 1999) using a gene-specific upstream primer (usst1A: 5'-CGCGCTACGGCG GACGGACTGT-3'; or usst1B: 5'-ACGGCGGTC CGACGGTAGCAAAG-3') and a downstream $t_{30}$ primer (identical to the $t_{30}$ region of the $d_{26} t_{30}$ primer: 5'-CATGTACGTTGATGAAGGGTGTC GTGGCAG-3'). Following an initial denaturation cycle of $94^{\circ} \mathrm{C}$ for $5 \mathrm{~min}, 39$ PCR cycles were performed; each cycle consisted of 1 min denaturation $\left(94^{\circ} \mathrm{C}\right), 1 \mathrm{~min}$ annealing $\left(65^{\circ} \mathrm{C}\right.$ for sst $1 \mathrm{~A}$ and $62{ }^{\circ} \mathrm{C}$ for sst $\left.1 \mathrm{~B}\right)$ and $1 \mathrm{~min}$ extension $\left(72{ }^{\circ} \mathrm{C}\right)$, except in the last cycle when the extension time was increased to $10 \mathrm{~min}$. The resulting RS-PCR products were subjected to Southern blot analysis using sst1A(as) or $\operatorname{sst1B}(\mathrm{as})$ gene-specific radiolabeled probes (Moore et al. 1999).

The amount of sst1A and sst1B mRNA in brain (optic tectum), pancreas and liver was measured using a quantitative slot-blot technique (Moore et al. 1999) in which in vitro-synthesized cRNA standards were blotted onto nylon membrane along with sample total RNA $(10 \mu \mathrm{g})$. The membranes were hybridized with radiolabeled $\operatorname{sst} 1 \mathrm{~A}(\mathrm{as})$ or $\operatorname{sst} 1 \mathrm{~B}(\mathrm{as})$ oligonucleotide probes, washed and visualized with the Packard Cyclone Imaging System. Sample blots were then stripped and rehybridized with a radiolabeled full-length $\gamma$-actin probe. Sample RNA abundance was calculated after correction for background and after $\gamma$-actin normalization.

\section{Data analyses}

Nucleotide and deduced amino acid sequences were aligned and analyzed with the GeneTool and PepTool sequence analysis programs (BioTools Inc., Edmonton, Alberta, Canada). ClustalX (default parameters) was used in conjunction with the neighbor-joining method (Saitou \& Nei 1987) to generate the phylogenetic tree; the tree was visualized with TreeView (Page 1996). Quantitative data are expressed as means \pm s.E.M. A two-tailed Student's $t$-test was used to estimate differences $(P<0 \cdot 05)$ between treatment groups (Sigma Stat, SPSS Inc., Chicago, IL, USA).

\section{Results}

\section{Characterization of two somatostatin receptor subtype 1 cDNAs}

Two distinct cDNA fragments, each approximately $500 \mathrm{bp}$ in size, were amplified by RT-PCR from total RNA isolated from the brain and Brockmann body (endocrine pancreas) of rainbow trout using two sst-specific primers (GSP-1 and GSP-2). These cDNAs (denoted fragment A and fragment $\mathrm{B}$ ) had 
sst1 Nucleotide Percent Identity

\begin{tabular}{|c|c|c|c|c|c|c|}
\hline Tsst1A & $63.6^{*}$ & 62.4 & 58.7 & 59.2 & 70.4 & 66.2 \\
\hline 94.1 & Tsst1B & 62.7 & 60.2 & 58.4 & 62.1 & 59.6 \\
\hline 62.4 & 62.9 & Gsst1A & 92.8 & 68.4 & 70.8 & 66.4 \\
\hline 62.1 & 62.4 & 98.6 & Gsst1B & 65.3 & 71.3 & 66.3 \\
\hline 64.4 & 65.5 & 77.7 & 77.1 & Hsst1 & 88.1 & 83.2 \\
\hline 63.9 & 65.0 & 77.1 & 76.6 & 98.7 & Msst1 & 96.1 \\
\hline 62.5 & 63.6 & 75.2 & 74.7 & 94.6 & 95.6 & Rsst1 \\
\hline
\end{tabular}

\section{sst1 Amino Acid Percent Identity}

Figure 7 Amino acid (lower left) and cDNA nucleotide (upper right) sequence identities of vertebrate sst1 subtypes. Percentage identities were calculated on sequences arranged for maximum alignment using the GeneTool 1.0 analysis program (BioTools, Inc.; Edmonton, Alberta, Canada). Sequences are obtained from: goldfish sst1A (Gsst1A) and sst1B (Gsst1B) (Lin et al. 1999a); human sst1 (Hsst1) and mouse sst1 (Msst1) (Yamada et al. 1992); rat sst1 (Rsst1) (Meyerhof et al. 1991); rainbow trout sst1A (Tsst1A) and rainbow trout sst1B (Tsst1B) (this study). ${ }^{*}$ When the extreme $5^{\prime}$ portion of sst1 $A$ and the extreme $3^{\prime}$ portion of sst1B is removed, nucleotide sequence identity between trout sst1A and sst1B is $88 \cdot 1 \%$.

nucleotide sequences similar to the coding region from TMD3 to TMD7 of mammalian ssts and were very similar in nucleotide sequence to each other. Further investigation using 3'-RACE PCR with GSP-3 (designed from the RT-PCR fragment obtained in phase I) revealed two distinct products, one $c .500 \mathrm{bp}$ and the other $c .850 \mathrm{bp}$. The $500 \mathrm{bp}$ product had an overlapping sequence with fragment A. The $850 \mathrm{bp}$ product had an overlapping sequence with fragment B. Reverse transcription using GSP-2 and 5'-RACE PCR with GSP-4 resulted in the amplification of two distinct fragments: one that was c. $950 \mathrm{bp}$ and had an overlapping sequence with fragment $\mathrm{A}$ and another that was c. $475 \mathrm{bp}$ and had an overlapping sequence with fragment B. Overlapping sequences of the $950 \mathrm{bp} \mathrm{5}$-RACE product, fragment $\mathrm{A}$, and the $500 \mathrm{bp}$ 3'-RACE product revealed a novel 1755 bp cDNA encoding a subtype 1 sst (designated sst1A; Fig. 1). This sequence contained a single initiation site 500 bases from the most $5^{\prime}$ end, and two potential putative polyadenylation sites: one 75 bases from the most $3^{\prime}$ end and the other 20 bases from the most $3^{\prime}$ end. Overlapping sequences of the $475 \mathrm{bp}$ 5'-RACE product, fragment B, and

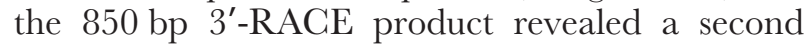
distinct 1743 bp cDNA which we designated sst1B (Fig. 1). This sequence contained a single initiation site 25 bases from the most $5^{\prime}$ end, and also contained two potential putative polyadenylation sites: one 465 bases from the most $3^{\prime}$ end and the other 18 bases from the most $3^{\prime}$ end.

The sstlA and sst1B cDNAs each contain a single open reading frame of $1113 \mathrm{bp}$ encoding a 371-amino acid receptor protein with seven hydrophobic TMDs, a feature found in the G-protein-coupled receptor family. They also contain the DRY motif at the border of TMD3 and the second intracellular loop, a sequence highly conserved in ssts as well as in other seven TMD receptors. The YANS.ANP.LY motif was also found in TMD7, a signature sequence in mammalian ssts (Fig. 1). The designation of these ssts as subtype 1 was based on similarity to other 
Ts 5 t 1 A $\mathrm{T} s \mathrm{st} 1 \mathrm{~B}$ Gs $s \mathrm{t} 1 \mathrm{~A}$

Gsst $1 \mathrm{~B}$ Hs: 1

Mast 1

Rsst 1

Tsat1 Tsst $1 \mathrm{~B}$ Gsst17 Gsst1B Hs $s$ t 1

Msst 1

Rsst 1

Ts st 1 A Tsst $1 \mathrm{~B}$ Gs:t1 GsatlB

Hsat 1

Msst 1

Rsst 1

Tsst 1 A Tsst1E Gsst1 GsatlB Hsst 1

Mast

Rsst 1

Tsst $1 \not$ Tsst1B GsstlA GsotlB Hsst 1

Mast 1

Rast 1

Tsst 17 TsEt $1 \mathrm{~B}$ Gsst $1 \mathrm{~A}$ GsatlB Hsst 1

Msst 1

Rsst 1

Ts st 1 A Ts 5 t $1 \mathrm{~B}$ Gsst1 Gstle

Hs $\mathrm{st} 1$

Msst 1

Rsst 1

M-DN- - - - - - - - - - MAANGTSNYPAYPTDFPYNSSIDYEDYDO-EPDT M-EN- - - - - - - - - - - MATNRSGDY FEYFTGLYNSSLDYEDYDQ-ELDA MLPNDTFK- - - - - - - - - - - - NLEDGLYLITFSSNETHNGD-SHGSMLPNDTFK- - - - - - - - - - - - - NLEDGLYLLNF SSNETHNGD-SHGSMF PNGTASSPSSSPSPSGSCGEGGGSRGPGAGAADGME--EPGRNASONGTLSEGOG MFPNGTASSPSSSPSPSPGSCGEGACSRGPGSGAADGME- -EPGRNASONGTLSEGQG MF FNGTAPSFTSSPSSS PGGCGEGVCSRGPGSGAADGME- - E PGRNSSONGTLSEGQG $* * * * * * * * * * * * * * * *$

TMD 1

TMD 2

SKI I I S IYALVCCVGLTGNTMVIYVI LKYAKMKTA TN IYI L NLA IADELFML SVPFI SKIIIPSIYALVCCWGLTGNAMVIYVI LKYAKMKTA TN I Y I NNLA IADELFMLSVPFI SAIFISFIYSVVCLVGLCGNSMVIYVI FRYAKMKTATNIYI LNLAIADELLML SVEFI SA IF ISF IYSVVCLVGLCGNSMVIYVIFRYAKMKTA TN IY I LNLA IADDLLMLSVPFI SAILISFIYSVVCLVGLCGNSMVIYVILRYAKMKTA TNIYILNLA IADELLML SVPFI SAILISFIYSVVCLVGLCGNSMVIYVI LRYAKMKTATNIYI LNLAIADELLML SVPFI SAILISFIYSVVCLVGLCGNSMVIYVILRYAKMKTATNIY I L TLA IADELLMLSVFFI

THD 3

ATSAAVRHWPFGSLMCRLVLSVDGI NMFTSIFCLTVLSVDRYVAVVHPIKAARYRRFT ATSAAVRHW FFGSLMCRLVLSVDGINMFTS IFCLTVLSVDRYVAVVHFIKAARYRRPT VTSSLLHHW PF GSLLCRLVLSVDA I NMFTS IYCLTVLS I RRYISVVHPI KAARYRRET VTSSLLHHW PFGSLLCRLVLSVDA INWFTS I YCLTVLS IDRY I SVVH P I KAARYRRPT VTSTLLRHW FFGALLCRLVLSVDAVNMFTS I YCLTVLSVQRYVAVVH P I KAARYRRPT VTSTLLRHWF FALLCRLVLSVDAVHMFTS IYCLTVLSVGRYVAVVHF I KAARYRRT VTSTLLRHW FFGALLCRLVLSVDAVNMFTS I YCLTVLSVPRYVAVVH I KAARYRRPT

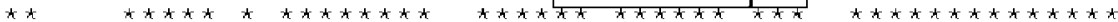

114

114

114

TMD 4

VAKVVNVCVWGLSLLVILAI I IFADTVPAODGGVDCNFLWPEAA- -WSEAFVVYTFLI VAKVVNVCVWGLSLLVILEII I FADTVEAODGGVDCNFLWEEAA- -WSVAFVVYTFLI I A KMVN LGVWMFS I LVI L F I I I FSTTA PNSDGSVACNMOWPE PEROWMAVFVI YAFLW I AKMVN LGVWMFS ILVILE I I IFSTTA PNSDGSVACNMOMEE PEROWMAVFVIYAFLM VAKVVNLGVWVLSLLVILF IVVFSRTAANSDGTVACNMLMPE PAORWLVGFVLYTFLM VAKVVNLGVWVLSLLVILF IVVFSRTAANSDGTVACNMLMPE PAORWLVGFVLYTFLM VAKVVNLGVWVLSLLVILF IVVFSRTAANSDGTVACNMLMEE RAORWLVGFVLITFLM

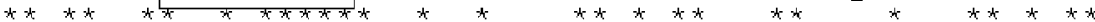

TMD 5

TMD 6

GFLLPVGAICLCYCLWVARMRAVGLKAGWLQRRRSEKKITRWVVCEVAVFVLCWWPFY GFLL FVGA ICLCYCLWVARMRA VGLKAGWLPRRRSEKK I TRMVLCIVAVFVLCWMPFY GF LF PVIA ICWCY I L I I VKWRVVA LKAGWOORKKSERK I TLWVMWVVTVFV I CWMPFH GF LL PVGA I CLCYVL I I AKMRMVA LKAGWOQRKRSERK I TLMVMMVVMVFV I CWMPFY GF LL PVGA ICLCYVL I I AKMRMVA LKAGWOQRKRSERK I TLMVMMVVMVFV I CWMPF Y GFLL FVGA I LCYVL I I AKMRMVA LKAGWOORKRSERK I TLMVMMVVMVFV I CWMFFY GELL PVGA ICLCYVL I IAKMRMVALKAGWOQRKRSERK I TLHVMTVVMVEV I CWMPFY

208 208 206 206 230 230 230

TMD 7

IVOLVSVFHHP PN PWVTOLFVI LSYANSGANPILYGFVSDNFRRSFORIVCFRWLESG I VOLVSVFHR P DAMVTOLFVI L $S Y A N S C A N P$ I L Y GFVS DNFRRSFOR I VCFRWLESG IMQLVSVFVOQHNATLSOLAVI LG YANSCANPI LYGFLSONFRRSFOR I LCLRWWDNA IVOLVSVFVOOHNSTLSOLAVI LGYANSCAN F I Y GFLSDNFRRSFOR I LCLRWWDNA VVOLVNVFAEODDATVSOL SVI LGYANSCAN F I L Y L LSDNF KRSFOR I LCLSWMDNA VVQLVNVFAEODDATVSOLSVILGYANSCANP I LYGF S DNFKRSFOR I LCLSWMDNA VVOLVNVFAEODDATVSOLSVI LGYANSCANF I L YGFLSDNEKRSFQR I LCLSWMDNA

LDCEOVDYRAVALKROATNGOKDFPKECLASDNVFRNGTYTSRTTTL LDGEOVDYRAVALKROATNGOKDF FKECLASGMVFONGTCTSRTTTI TE-EFIDYYATALKSRGYSVD-DFOFENLESGSTYRNGTCTSRTTTL TE-EPIDYYATALKSRGYSVD-DFOFDNLESGSTYRNGTCTSRTTTI AE-EPVDYYATALKSRAYSVE-DFOFENLESGGVFRNGTCTSRITTL AE-E FVDYYATALKSRAYSVE-DFOFENLESGGVFRNGTCASRISTI AE-EFVDYYATALKSRAYSVE-DFQFENLESGGVFRNGTCASRISTL

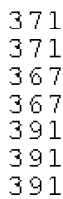

371

367

391

391 
known sst sequences. Rainbow trout sst1A and sst1B share $63 \cdot 6 \%$ nucleotide identity and $94 \cdot 1 \%$ amino acid identity.

Despite the similarity of sequence between rainbow trout sstlA and sst $1 \mathrm{~B}$, we took advantage of two regions of the nucleotide sequence within the 500 bp RT-PCR fragments to design oligonucleotide probes that would distinguish between the two transcripts. Southern blot analysis of positive size-fractionated sstl PCR products from phase I RT-PCR showed specific binding of these radiolabeled oligonucleotide probes to the RTPCR fragments; the sstlA-specific probe only hybridized to sst1A cDNA (fragment A), the sst1B-specific probe only hybridized to sst1B cDNA (fragment B) and neither probe hybridized to a partial rainbow trout sst2 cDNA (data not shown). Northern blot analysis of size-fractionated poly $(\mathrm{A})^{+}$ RNA from brain and pancreas revealed that there appears to be a single transcript encoding sstlA and a single transcript encoding sstlB (Fig. 2).

\section{Differential expression of sst1 mRNAs among tissues}

RNA from various tissues was extracted and reverse transcribed. The resulting cDNAs encoding sstlA and sstlB were amplified by RS-PCR, electrophoresed and subjected to Southern blot analysis using the sst1A(as) or sst $1 \mathrm{~B}(\mathrm{as})$ radiolabeled nucleotide probes. Using this method, rainbow trout sst1A was found in brain (whole), stomach, liver, pancreas, upper and lower intestine, pyloric cecum, kidney and muscle (Fig. 3A). In addition to the tissues in which sst1A mRNA was found, sst1B mRNA was also found in the esophagus (Fig. 3B). Duplicate samples pretreated with RNase demonstrated that amplified products were exclusively obtained from RNA templates and not false positives originating from contaminating genomic DNA. The sizes of the amplified cDNAs for sstlA and sst1B from RS-PGR were 494 and $486 \mathrm{bp}$ respectively, and matched the sizes predicted from primer locations on the cDNA sequences.
Northern slot-blot analysis showed that sstlA and sst1B mRNA were uniformly distributed among the major regions of the trout brain (Fig. 4).

\section{Differential abundance of sst1 mRNAs within tissues}

Hybridization of gene-specific oligonucleotide probes to replicate slot-blots containing known quantities of in vitro-synthesized sstlA and sst1B cRNA standards (in the range $1.5 \times 10^{7}-1.5 \times 10^{9}$ molecules) and RNA extracted from selected tissues allowed for accurate evaluation of the amounts of sst1A and sst1B mRNAs (Fig. 5; note the lack of cross-hybridization). This approach was used to determine the abundance of sst1A and sstlB mRNAs in the optic tectum, pancreas and liver (tissues that play central roles in the coordination of growth, development and metabolism) removed from rainbow trout under normal physiological conditions (Fig. 6). Under these conditions, levels of sstlA mRNA were significantly higher (16-fold) than those of sst1B in the optic tectum. In contrast, sst1B mRNA levels were significantly higher (4-fold) than those of sstlA in the liver. No difference between the levels of sstlA mRNA and sst1B mRNA were detected in the pancreas.

\section{Discussion}

In this study, we characterized two cDNAs that encode for distinct type 1 SS receptors from the endocrine brain and endocrine pancreas of rainbow trout, designated sstlA and sst1B. The nucleotide identity between the cDNAs is $63.6 \%$ $\left(88 \cdot 1 \%\right.$ when the $5^{\prime}$ and $3^{\prime}$ untranslated regions are excluded); the position and extent of the differences suggests the existence of two non-allelic sst 1 genes. We also demonstrate for the first time to our knowledge that the mRNAs encoding sst isoforms are differentially expressed, both in terms of distribution among tissues as well as in terms of abundance within tissues.

Figure 8 Comparison of amino acid sequences of rainbow trout sst1A and sst1B with those of: goldfish sst1 $\mathrm{A}$ and sst1B (Gsst1A and Gsst1B) (Lin et al. 1999a); human sst1 (Hsst1) and mouse sst1 (Msst1) (Yamada et al. 1992); rat sst1 (Rsst1) (Meyerhof et al. 1991). Sequence identity was maximized by inserting gaps (dashed lines). Conserved amino acid residues are indicated by asterisks. The putative TMDs are overlined. Notable structural motifs are boxed. 
To date, molecular cloning has revealed the existence of five subtypes of somatostatin receptor (sst1-sst5) in several mammalian (Hoyer et al. 1995, Reisine \& Bell 1995, Patel \& Srikant 1997, Meyerhof 1998) and non-mammalian species (Lin et al. 1999a, 2000, 2002, Zupanc et al. 1999, Bossis \& Porter 2001, Lin \& Peter 2003). Somatostatin receptor subtype 1 has been found in human, mouse (Yamada et al. 1992) and rat (Meyerhof et al. 1991) as well as two isoforms in goldfish (Lin et al. 1999a). The characterization of trout ssts contributes further to our understanding of the evolution of the sst family and provides new insight into structure-function relationships of the SS signaling systems (cf. Sheridan et al. 2000). When compared with nucleotide sequences from other cloned sst1 subtypes, rainbow trout sstlA is most homologous to that of mouse sstl with $70.4 \%$ similarity (Fig. 7; Yamada et al. 1992). Rainbow trout sstlB on the other hand is most similar to goldfish sst1A, sharing 62.7\% nucleotide identity (Fig. 7; Lin et al. 1999a). Even lower is the degree to which rainbow trout sst1A and sst1B share similarities with other sst subtypes, i.e. sst2-sst5 (data not shown). As expected, the largest number of differences between the nucleotide sequences occurs in the untranslated regions of the cDNAs.

The deduced sst1A and sst1B proteins produced in rainbow trout islet cells and brain tissue share $94 \cdot 1 \%$ amino acid identity and both contain 371 amino acids (Figs 7 and 8), slightly longer than the 367-amino-acid proteins in goldfish sst1A and sst1B (Lin et al. 1999a), but shorter than the 391-aminoacid proteins in human and mouse sstl (Yamada et al. 1992) and rat sstl (Meyerhof et al. 1991). The trout sst $1 \mathrm{~A}$ and sst1B deduced amino sequences are most similar to human sstl, sharing $64 \cdot 4$ and $65 \cdot 5 \%$ identity respectively (Figs 7 and 8 ). Rainbow trout sstlA and sst1B share only $43-60 \%$ amino acid identity with mammalian sst2-sst5 (data not shown).

Structurally, the deduced amino acid sequences of rainbow trout sstlA and sstlB share many similarities with the other cloned vertebrate sstls. The extracellular N-terminus and the intracellular C-terminus of trout sstlA and sst1B account for the greatest number of differences when compared with other vertebrate sstls, whereas the TMDs and the intra- and extracellular loops are highly conserved. Several conserved sequence patterns are found in the trout sstls which are also found in other sstls as well as other sst subtypes. Many members of the DRY-containing family of G-protein-coupled receptors also contain these conserved motifs. These amino acid motifs may play a role in conferring the appropriate tertiary structure necessary for functional activity and include the motifs GN..V, NLA.AD, S...L...S.DRY, W..S....P, F..P, F..GW.P and NS..NP..Y in $\alpha$-helical TMDs 1-7 respectively (Reisine \& Bell 1995). The search for structural motifs that are specific for the sst family has revealed a putative phosphorylation site R..SE in the third intracellular loop and the motif L.YANS.ANP.LY.F.S in TMD7, both of which are found in the trout sstls (Fig. 8).

Believed to be glycoproteins (Rens-Domiano \& Reisine 1991, 1992), ssts contain consensus sequences in the $\mathrm{N}$-terminal domain of the receptors with the sequence Asn-X-Ser/Thr (N-X$\mathrm{S} / \mathrm{T}$ ), where $\mathrm{X}$ is any amino acid except proline. Trout sstlA and sstlB contained two potential Asn-linked glycosylation sites at amino acid positions $\mathrm{Asn}^{7}$ and $\mathrm{Asn}^{22}$. The nature and extent to which receptors are glycosylated may play a role in high-affinity ligand binding (Rens-Domiano \& Reisine 1992). Both Asn-linked glycosylation sites were either identical or similar in sequence to the corresponding site in human, rat, mouse and goldfish sst1s (Fig. 8; Meyerhof et al. 1991, Li et al. 1992, Yamada et al. 1992, Lin et al. 1999a).

Another structural feature found in the rainbow trout sstls is two conserved cysteine residues (Fig. 8). These are found at positions Cys ${ }^{110}$ and Cys ${ }^{188}$ in the first and second putative extracellular loops respectively, and are thought to be associated with the formation of a disulfide bridge that would stabilize the tertiary structure of the receptor as seen in other G-protein-coupled receptors (Strader et al. 1994).

Three potential amino acids were found which could be cAMP-dependent protein kinase phosphorylation sites at positions $\mathrm{Thr}^{152}, \mathrm{Ser}^{243}$ and $\mathrm{Thr}^{248}$ for both sst1A and sst1B. In addition, potential protein kinase $\mathrm{C}$ phosphorylation sites were found at position $\mathrm{Thr}^{365}$ for sstlA and position Thr $^{365}$ for sst1B (Fig. 8; Kemp \& Pearson 1990).

An additional highly conserved amino acid residue at position Cys ${ }^{317}$, which is found in the C-terminus, has been shown to be palmitoylated in $\beta_{2^{-}}$and $\alpha_{2}$-adrenergic receptors as well as the 


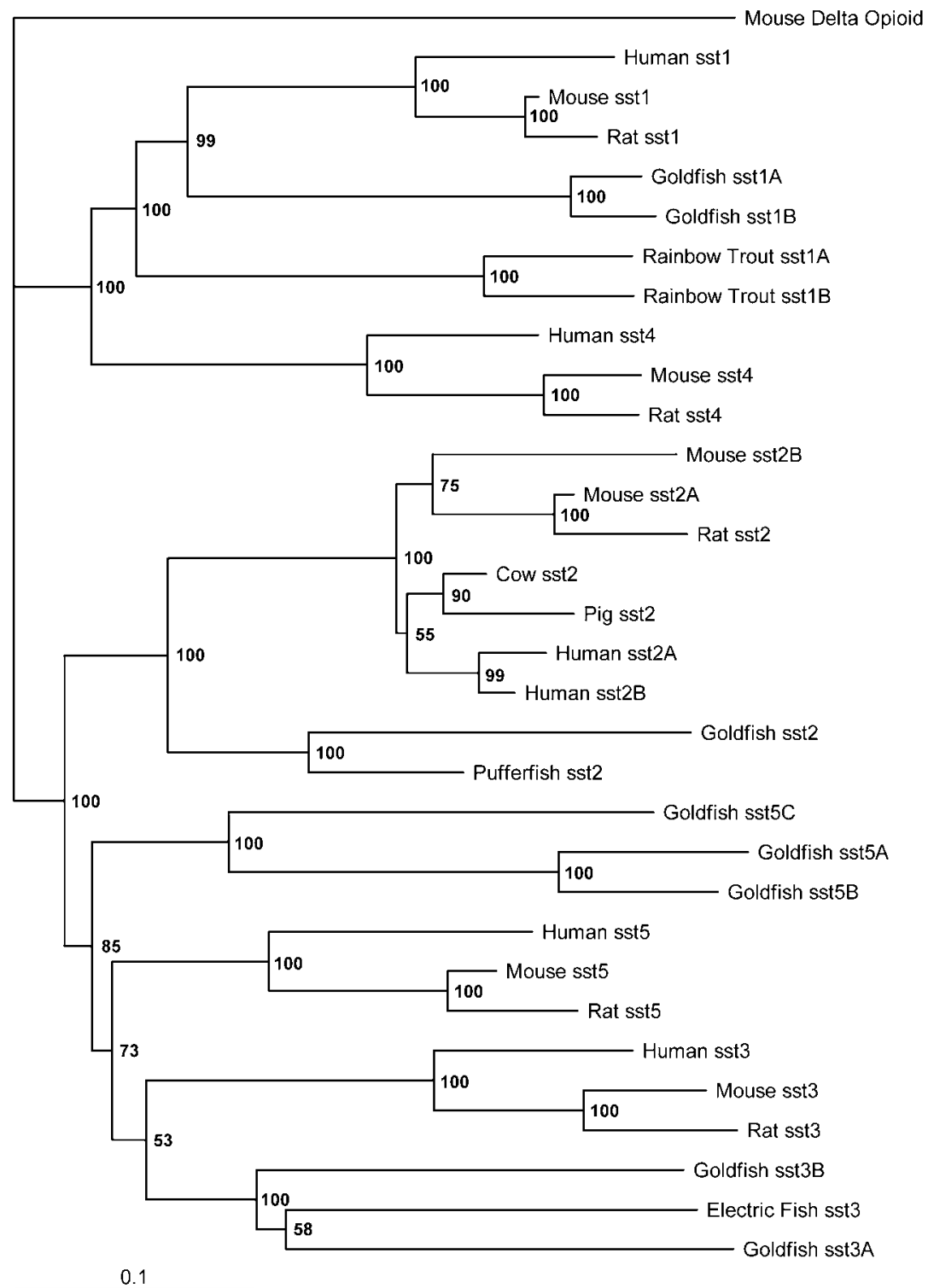

Figure 9 Phylogenetic tree based on the alignment of amino acid sequences of known somatostatin receptors in vertebrates. The branch 'lengths' represent amino acid substitutions per site from a common ancestor and are proportional to the estimated elapsed time since the divergence occurred. The bold numbers at each branch point represent the bootstrap values, scaled to a maximum of $100 \%$. The following sequences were obtained from GenBank: mouse opioid receptor, AF062753; human sst1, M81829; human sst2A and human sst2B, AF18174, human sst3, M96738; human sst4, D16826; human sst5, D16827; mouse sst1, M81831; mouse sst2A, M81832; mouse sst2B, X68951; mouse sst3, M91000; mouse sst4, U26176; mouse sst5, U82697; rat sst1, M97656; rat sst2, M93273; rat sst3, X63574; rat sst4, U04738; rat sst5, L04535; pig sst2, D21338; cow sst2, L06613; goldfish sst1A, AF097726; goldfish sst1B, AF097727; goldfish sst2, AF139597; goldfish sst3, AF311307; goldfish sst5, AF252879; pufferfish sst 2, AF329947. The electric fish (Apteronotus) sst3 sequence is from Zupanc et al. (1999). 
rhodopsin family of receptors and could be used to anchor the C-terminal end of the receptor to the plasma membrane (Fig. 8; O'Dowd et al. 1989, Papac et al. 1992, Kennedy \& Limbird 1993).

Other important features of the sstl receptors are conserved amino acids that may play a role in the binding of the ligand SS-14. Kaupmann et al. (1995) proposed a model based upon site-directed mutagenesis studies of different ssts. These findings show that the core residues of SS-14, Phe ${ }^{6}$-PheTrp-Lys-Thr-Phe ${ }^{11}$, interact with a ligand-binding pocket located in TMDs $3-7$, which is lined with hydrophobic and charged amino acids. In trout sstls, the ligand-binding pocket is lined by $\mathrm{Phe}^{265}$ and Gln $^{269}$ in TMD6 and by Thr ${ }^{283}$ in TMD7 (Fig. 8). This pocket may provide a hydrophobic environment for interaction with the $\mathrm{Phe}^{6}-\mathrm{Phe}^{11}$ region of SS-14; this interaction may be enhanced by an electrostatic interaction between Lys $^{9}$ of the ligand and Asp ${ }^{117}$ of TMD3 (Sheridan et al. 2000).

The phylogenetic relationships of the cloned trout sst1s with other known members of the sst family is shown in Fig. 9. The pattern that emerges supports a multigenic origin of ssts that arose from a series of gene duplication events throughout the course of vertebrate evolution. Two major clades can be identified: an sstl/sst4 group and an sst2/sst3/sst5 group. Subtype 1 ssts appear to have diverged early from $\mathrm{sst} 4 \mathrm{~s}$, similar to the divergence of sst2s from sst5/sst3s. The branching of the sst1 clade is consistent with the phylogeny of vertebrates, with rainbow trout representing one of the oldest species so far examined. The emergence of sstl isoforms in fish, both rainbow trout and goldfish (Lin et al. 1999a), probably results from a recent duplication event such as the tetraploidization of these species (Ohno 1970). The existence of sst isoforms in other tetraploid or non-tetraploid species is not known. The significance of different sst isoforms is not clear, but they may play a role in selective binding of SSs that circulate in fish (e.g. SS-14, $\left.\left[\mathrm{Tyr}^{-{ }^{7}}{ }^{,} \mathrm{Gly}^{10}\right]-\mathrm{SS}-14, \quad\left[\mathrm{Tyr}^{7}, \mathrm{Gly}^{10}\right]-\mathrm{SS}-25\right)$ or in activating specific biological responses.

The two sst $1 \mathrm{mRNAs}$ are differentially expressed. This is supported by several observations. First, the expression pattern of sstlA and sstlB is tissue specific. While sstlA and sst1B mRNAs are distributed widely in the brain, pancreas, kidney and selected regions of the gut, only sst1B mRNA was detected in the esophagus; sstl has also been found broadly distributed in mammals (Sheridan et al. 2000). Lin et al. (1999a) also reported a pattern of tissue-specific expression for sstl isoforms in goldfish. Secondly, the abundance of the rainbow trout sstl mRNAs was different within specific tissues. Within the optic tectum, the predominant form was that encoding for sstlA, whereas in the liver, sst1B mRNA was the predominant form. There appeared to be no difference in the abundance of sstlA and sstlB mRNAs in the pancreas. These results suggest the production of two forms of sstl in trout and that there may be independent mechanisms which regulate the expression of each variant. The factors which serve to regulate sst expression remain to be investigated, but likely candidates include factors from systems that are known to be affected by SS (e.g. growth hormone and insulin).

\section{Acknowledgements}

We are grateful to Jeff Kittilson, Alexio Santiago, Brendan Kelly and Matt Friedt for their technical assistance.

\section{Funding}

This research was supported by grants from the National Science Foundation, USA (IBN 0076416, EPS 0132289) to M A S. No conflict of interest exists that would prejudice the impartiality of the research.

\section{References}

Ahn SC, Baek BS, Oh T, Song CS \& Chatterjee B 2000 Rapid mini-scale plasmid isolation for DNA sequencing and restriction mapping. Biotechniques 29 466-468.

Bossis I \& Porter TE 2001 Identification of the somatostatin receptor subtypes involved in regulation of growth hormone secretion in chickens. Molecular and Cellular Endocrinology 182 203-213.

Conlon J M 1989 Biosynthesis of regulatory peptides - evolutionary perspectives. In The Comparative Physiology of Regulatory Peptides, pp 174 202. Ed. S Holmgren. London/New York: Chapman \& Hall. Conlon JM, Tostivint H \& Vaudry H 1997 Somatostatin- and urotensin II-related peptides: molecular diversity and evolutionary perspectives. Regulatory Peptides 69 95-103.

Gunning P, Ponte P, Okayama H, Engel J, Blau H \& Kedes L 1983 Isolatcterization of full-length cDNA clones for $\alpha$-, $\beta$-, and $\gamma$-actin mRNAs; skeletal but not cytoplasmic actins have amino-terminal cysteine that is subsequently removed. Molecular and Cellular Biology $3787-795$.

Hoyer D, Bell GI, Berelowitz M, Epelbaum J, Feniuk W, Humphrey PPA, O'Carroll AM, Patel YG, Schonbrunn A, Taylor JE \& Reisine T 1995 Classification and nomenclature of somatostatin receptors. Trends in Pharmacological Sciences 16 86-88. 
Kaupmann K, Bruns C \& Raulf R 1995 Two amino acids, located in transmembrane domains VI and VII, determine the selectivity of the peptide agonist SMS 201-995 for the SSTR2 somatostatin receptor. EMBO Fournal 14 727-735.

Kemp BE \& Pearson RB 1990 Protein kinase recognition motifs. Trends in Biochemical Sciences 15 342-346.

Kennedy M \& Limbird LE 1993 Mutations of the $\alpha_{2 \mathrm{~A}}$-adrenergic receptor that eliminate detectable palmitoylation do not perturb receptor-G-protein coupling. Fournal of Biological Chemistry 268 8003-8011.

Kittilson JD, Moore CA \& Sheridan MA 1999 Polygenic expression of somatostatin in rainbow trout: evidence of a preprosomatostatin encoding somatostatin-14. General and Comparative Endocrinology 114 $88-96$.

Li XJ, Forte M, North RA, Ross CA \& Snyder SH 1992 Cloning and expression of a rat somatostatin receptor enriched in brain. Fournal of Biological Chemistry 267 21307-21312.

Lin X \& Peter RE 2001 Somatostatins and their receptors in fish. Comparative Biochemistry and Physiology B 129 543-550.

Lin X \& Peter RE 2003 Somatostatin-like receptors in goldfish: cloning of four new receptors. Peptides 24 53-63.

Lin X, Janovick JA, Brothers S, Conn PM \& Peter RE 1999a Molecular cloning and expression of two type one somatostatin receptors in goldfish brain. Endocrinology $1405211-5219$.

Lin X, Otto CJ \& Peter RE 1999 b Expression of three distinct somatostatin messenger ribonucleic acids (mRNAs) in goldfish brain: Characterization of the complementary deoxyribonucleic acids, distribution and seasonal variation of the mRNAs, and action of a somatostatin-14 variant. Endocrinology 140 2089-2099.

Lin X, Janovick JA, Cardenas R, Conn PM \& Peter RE 2000 Molecular cloning and expression of a type-two somatostatin receptor in goldfish brain and pituitary. Molecular and Cellular Endocrinology 166 75-87.

Lin X, Nunn C, Hoyer D, Rivier J \& Peter RE 2002 Identification and characterization of a type five-like somatostatin receptor in goldfish pituitary. Molecular and Cellular Endocrinology 189 105-116.

Meyerhof W 1998 The elucidation of somatostatin receptor functions: a current view. Reviews in Physiology Biochemistry and Pharmacology 133 55-108.

Meyerhof W, Paust HJ, Schonrock C \& Richter D 1991 Cloning of a cDNA encoding a novel putative G-protein-coupled receptor expressed in specific rat brain regions. DNA Cell Biology 10 689-694.

Moore CA, Kittilson JD, Ehrman MM \& Sheridan MA 1999 Rainbow trout (Oncorhynchus mykiss) possess two somatostatin mRNAs that are differentially expressed. American Fournal of Physiology 246 R1553-R1561.

O'Dowd BF, Hnatowich M, Caron MG, Lefkowitz RJ \& Bouvier M 1989 Palmitoylation of the human $\beta_{2}$-adrenergic receptor. Mutation of Cys341 in the carboxyl tail leads to an uncoupled nonpalmitoylated form of the receptor. Fournal of Biological Chemistry $2647564-7569$.

Ohno S 1970 Evolution by Gene Duplication. Berlin: Springer-Verlag.

Page RDM 1996 TreeView: An application to display phylogenetic trees on personal computers. Computer Applications in the Biosciences: CABIOS 12 357-358.

Papac DI, Thornburg KR, Bullesbach EE, Crouch RK \& Knapp DR 1992 Palmitoylation of a G-protein coupled receptor. Direct analysis by tandem mass spectrometry. Fournal of Biological Chemistry 267 16889-16894.

Patel YC \& Srikant CB 1997 Somatostatin receptors. Trends in Endocrinology and Metabolism 8 398-405.

Pesek MJ \& Sheridan MA 1996 Fasting alters somatostatin binding to liver membranes of rainbow trout (Oncorhynchus mykiss). Fournal of Endocrinology 150 179-186.

Reisine T \& Bell GI 1995 Molecular biology of somatostatin receptors. Endocrine Reviews 16 427-442.

Rens-Domiano S \& Reisine T 1991 Structural analysis and functional role of the carbohydrate component of rat brain somatostatin receptors. Fournal of Biological Chemistry $\mathbf{2 6 6}$ 20094-20102.

Rens-Domiano S \& Reisine T 1992 Biochemical and functional properties of somatostatin receptors. Fournal of Neurochemistry $\mathbf{5 8}$ 1987-1996.

Saitou N \& Nei M 1987 The neighbor-joining method: a new method for reconstructing phylogenetic trees. Molecular Biology and Evolution 4 406-425.

Sambrook J, Fritsch EF \& Maniatis T 1989 Molecular Cloning: A Laboratory Manual, edn 2. Plainview, NY: Cold Spring Harbor Laboratory Press.

Sheridan MA, Kittilson JD \& Slagter BJ 2000 Structure-function relationships of the signaling system for the somatostatin peptide hormone family. American Zoologist 40 269-286.

Shuldiner AR, Tanner K, Moore CA \& Roth J 1991 RNA template-specific PCR (RS-PCR): an improved method that dramatically reduces false positives in RT-PCR. Biotechniques $\mathbf{1 1}$ 760-763.

Strader CD, Fong TM, Tota MR \& Underwood D 1994 Structure and function of $\mathrm{G}$ protein-coupled receptors. Annual Reviews in Biochemistry 63 101-132.

Yamada Y, Post SR, Wang K, Tager HS, Bell GI \& Seino S 1992 Cloning and functional characterization of a family of human and mouse somatostatin receptors expressed in brain, gastrointestinal tract, and kidney. PNAS 89 251-255.

Zupanc GKH, Siehler S, Jones EMC, Seuwen K, Furuta H, Hoyer D \& Yano H 1999 Molecular cloning and pharmacological characterization of a somatostatin receptor subtype in the gymnotiform fish Apteronotus albifrons. General and Comparative Endocrinology 15 333-345.

Received 8 September 2003 Accepted 10 November 2003 